\title{
Molecular Characterization of Heterologous HIV-1gp120 Gene Expression Disruption in Mycobacterium bovis BCG Host Strain: A Critical Issue for Engineering Mycobacterial Based-Vaccine Vectors
}

\author{
Joan Joseph, ${ }^{1}$ Raquel Fernández-Lloris, ${ }^{1}$ Elías Pezzat, ${ }^{1,2}$ Narcís Saubi, ${ }^{1}$ Pere-Joan Cardona, ${ }^{3}$ \\ Beatriz Mothe, ${ }^{4}$ and Josep Maria Gatell ${ }^{1}$ \\ ${ }^{1}$ AIDS Research Unit, Hospital Clinic/IDIBAPS-HIVACAT, University of Barcelona, Calle Villarroel 170, 08036, Barcelona, Spain \\ ${ }^{2}$ Facultad de Medicina de la Benemérita, Universidad Autónoma de Puebla, Calle 13 Sur 2702, Puebla, 7200, Mexico \\ ${ }^{3}$ Unitat Tuberculosi Experimental, Institut "Germans Trias i Pujol”, Carretera del Canyet S/N, Badalona, 08916, Barcelona, Spain \\ ${ }^{4}$ AIDS Research Institute IrsiCaixa-HIVACAT, Universitat Autònoma de Barcelona, Hospital Germans Trias i Pujol, \\ Carretera del Canyet S/N, Badalona, 08916, Barcelona, Spain \\ Correspondence should be addressed to Joan Joseph, jjoseph@clinic.ub.es
}

Received 14 January 2010; Revised 26 March 2010; Accepted 22 April 2010

Academic Editor: Guihua H. Bai

Copyright () 2010 Joan Joseph et al. This is an open access article distributed under the Creative Commons Attribution License, which permits unrestricted use, distribution, and reproduction in any medium, provided the original work is properly cited.

Mycobacterium bovis Bacillus Calmette-Guérin (BCG) as a live vector of recombinant bacterial vaccine is a promising system to be used. In this study, we evaluate the disrupted expression of heterologous HIV-1gp120 gene in BCG Pasteur host strain using replicative vectors pMV261 and pJH222. pJH222 carries a lysine complementing gene in BCG lysine auxotrophs. The HIV-1 gp120 gene expression was regulated by BCG hsp60 promoter (in plasmid pMV261) and Mycobacteria spp. $\alpha$-antigen promoter (in plasmid pJH222). Among 14 rBCG:HIV-1gp120 (pMV261) colonies screened, 12 showed a partial deletion and two showed a complete deletion. However, deletion was not observed in all 10 rBCG:HIV-1gp120 (pJH222) colonies screened. In this study, we demonstrated that E. coli/Mycobacterial expression vectors bearing a weak promoter and lysine complementing gene in a recombinant lysine auxotroph of BCG could prevent genetic rearrangements and disruption of HIV 1gp120 gene expression, a key issue for engineering Mycobacterial based vaccine vectors.

\section{Introduction}

The need for a safe and effective human immunodeficiency virus (HIV) vaccine has never been greater. The acquired immune deficiency syndrome (AIDS) epidemic update released by UNAIDS on December 2009, documented that the overall number of people with HIV has stabilized, albeit at an unacceptably high level of about 33.4 million people living with HIV and 2.7 million individuals being newly infected with the virus in 2008. The global AIDS epidemics killed 2 million people in 2008, and the number of children orphaned by AIDS may reach 25 millions by 2010 . About $85 \%$ of these new infections occur in developing countries where access to antiretrovirals is still an enormous challenge $[1,2]$.
There is strong evidence to support a role of cytotoxic $\mathrm{T}$ lymphocytes (CTLs) in the containment of HIV replication [3-6] and several vaccine approaches have been pursued to elicit anti-HIV CTL responses [7]. One promising approach is to use Mycobacterium bovis Bacillus CalmetteGuérin (BCG) as a live recombinant bacterial vaccine vector. BCG vaccine has been used to immunize more than two billion individuals against tuberculosis with a long record of effectiveness and safety for use in humans [8]. CTL induction against HIV-1 and simian immunodeficiency virus (SIV) gag or env antigens has been described following the immunization of mice or nonhuman primates with recombinant $\mathrm{BCG}(\mathrm{rBCG})$ expressing these antigens $[8,9]$. More recently, recombinant Mycobacterium bovis Bacillus Calmette-Guérin (BCG) expressing HIVA immunogen has 
been generated and shown to be stable and to induce durable and high-quality HIV-1-specific CD4+ and CD8+ $\mathrm{T}$-cell responses in $\mathrm{BALB} / \mathrm{c}$ mice. Furthermore, when the recombinant BCG vaccine was used in a priming-boosting regimen with heterologous components, the HIV-1-specific responses provided protection against surrogate virus challenge, and the recombinant BCG vaccine alone protected against aerosol challenge with $M$. tuberculosis [10].

Three issues are critical for engineering a stable and immunogenic mycobacterial-based vaccine vector: (i) antigen localization, (ii) codon optimization, and (iii) in vivo plasmid DNA stability and genetic rearrangements. The antigen secretion and fusion of foreign antigens to mycobacterial surface lipoproteins will provide these antigens with access to the major histocompatibility complex (MHC) class I pathway and subsequently enhance the immunogenicity and to prevent foreign proteins from becoming toxic to BCG [11]. The use of mycobacterial optimal codons will enhance the transcriptional/translational activity of the foreign gene. The plasmid stability in vivo is essential for heterologous gene expression [8].

Genetic rearrangements have been described occurring in eukaryotes and prokaryotes [12]. Homologous recombination, which takes place between repeated DNA sequences, is one of the most important mechanisms for bacterial genome rearrangements. Any repeated DNA sequences on a chromosome can induce homologous recombination, but insertion sequences (IS) are among the most abundant of these [13-15]. Mycobacteria have been reported to contain several IS. In Mycobacterium tuberculosis, it has been suggested that IS6110 is an important source of genome variation. However, the role of such IS elements has not been thoroughly explored in heterologous gene expression in mycobacteria [16-18].

Expression of heterologous antigen in recombinant BCG necessarily imposes a metabolic burden. The extent of this burden will determine the degree to which fitness of the recombinant BCG is compromised. Thus, the relative level of fitness of the recombinant and any derived mutants will determine the rate at which the inserted element (structural instability) or its expression (functional instability) is lost from the bacterial population [19].

Husson et al. constructed a plasmid shuttle vector that allows insertion of foreign DNA and stable integration in the mycobacterial genome by homologous recombination. This shuttle vector was developed to express foreign antigens in $M$. smegmatis [20]. The structural instability of several recombinant plasmids expressing the cirscumsporozoite protein (CSP aa 18-391) from Plasmodium falciparum was described by Haeseleer [21]. The analysis in M. smegmatis and BCG of numerous rearranged plasmids showed that the presence of a functional specific expression cassette was responsible for the plasmid instability and that the observed deletions seemed to come from recombination between homologous and nonhomologous sequences [21].

Kumar et al. monitored the role of such genetic rearrangement events in the expression of cloned Escherichia coli lacZ gene in Mycobacterium smegmatis host strain using integrative (pMV361::lacZ) and episomal (pMV261::lacZ) vectors. The lac $Z$ gene present in both vectors was the mutable target within the vector and simple color detection assay in mycobacteria was used to screen the genetic rearrangements during replication in Mycobacterium smegmatis. They reported that a loss of lacZ phenotype was due to the insertion of an IS element in the lac $Z$ gene of the integrative vector (frequency $1.7 \times 10^{-5}$ ), whereas in the case of the replicative vector, the loss of lac $Z$ phenotype was due to deletions of different sizes in the lac $Z$ gene and the hsp60 promoter region (frequency $2 \times 10^{-3}$ ) [22].

In this study we evaluated the expression of disrupted heterologous HIV-1gp120 gene, from SHIV-HXBc2P 3.2 clone (GenBank accession number AF041850), by genetic rearrangements in Mycobacterium bovis BCG host strain using a replicative vector (pMV261) regulated by BCG hsp60 promoter (strong promoter). To compare the HIV1gp120 gene expression and plasmid DNA stability in vivo, the replicative (pJH222) and integrative ( $\mathrm{pJH} 223)$ vectors carrying a wild-type lysine-complementing gene in the lysine auxotroph of BCG host strain were used. In these vectors, the HIV-1gp120 gene expression was regulated by Mycobacteria $s p p$. $\alpha$-antigen promoter (weak promoter). We have demonstrated that the use of weak promoters (Mycobacteria spp. $\alpha$ antigen promoter) to regulate HIV-1gp120 gene expression and BCG lysine auxotrophs complemented with a lysine gene do, in fact, prevent the disruption of gene expression caused by genetic rearrangements.

\section{Materials and Methods}

2.1. Bacterial Strains and Culture Methods. The bacterial strains used in this study are listed in Table 1. Escherichia coli cultures were grown in Luria-Bertani (LB) broth (SigmaAldrich, USA) or on LB agar plates (Sigma-Aldrich, USA) at $37^{\circ} \mathrm{C}$. LB was supplemented with kanamycin $(40 \mu \mathrm{g} / \mathrm{mL})$ (Sigma-Aldrich, USA). Mycobacterial cultures were grown in Middlebrook 7H9 broth (Becton-Dickinson, USA) or on Middlebrook 7H10 agar medium (Becton-Dickinson, USA) supplemented with $10 \%$ albumin-dextrose-catalase (ADC) (Becton-Dickinson, USA) and containing 0.05\% Tween 80 (Sigma-Aldrich, USA) and kanamycin $(25 \mu \mathrm{g} / \mathrm{mL})$. The L-lysine monohydrochloride was purchased from SigmaAldrich (USA), dissolved in distilled water and used at a concentration of $40 \mu \mathrm{g} / \mathrm{mL}$.

2.2. Construction of Expression Vectors. Tables 2 and 3 listed the parental E. coli/mycobacterial shuttle vectors used for cloning the HIV-1gp120 gene from SHIV-HXBc2P 3.2 clone (GenBank accession number AF041850) and the constructs. Plasmid DNA vectors pMV261 (4,480 bp), pJH222 (6,423 bp), and pJH223 (6,313 bp) were used as parental plasmids for all plasmid DNA constructs obtained as described below. The entire DNA coding sequence of HIV1gp120 (1,578 bp) antigen was synthesized by polymerase chain reaction (PCR), using oligonucleotide primers specific for HIV-1 gp120 gene and cloned into the different E.coli/mycobacterial shuttle vectors. 
TABLE 1: List of bacterial strains used in this study.

\begin{tabular}{llr}
\hline Bacterial strains & Relevant characteristics & Reference or source \\
\hline Mycobacterium bovis BCG & 1173 P2 Pasteur strain & [23] \\
Mycobacterium bovis BCG mc ${ }^{2} 1604$ & Pasteur $\Delta$ lysA5::res & [24] \\
Escherichia coli JM109 & recA1 endA1 gyrA96 thi-1 hsdR17 $\left(\mathrm{rK}^{-} \mathrm{mk}^{+}\right)$supE44 & GIBCO BRL \\
\hline
\end{tabular}

TABLE 2: List of parental E. coli/mycobacterial shuttle vectors used in this study.

\begin{tabular}{lcc}
\hline Plasmid DNAs & Relevant characteristics & Reference or source \\
\hline pMV261 & E. coli/mycobacterial shuttle vector & \\
& Multicopy extrachromosomal vector & \\
& Kanamycin resistant & \\
pJH22 & pMV261 derivative & \\
& Multicopy extrachromosomal vector & Kindly provided by Bloom and Jacobs laboratory \\
& Kanamycin resistant & \\
PJH223 & pMV361 derivative & \\
& Monocopy integrative vector & \\
& Kanamycin resistant & \\
\end{tabular}

Plasmid DNA pMV261 is a replicative vector (multicopy, extrachromosomal) that contains a DNA cassette encoding kanamycin resistance (Tn903-derived aph gene), an E. coli origin of replication (oriE), a mycobacterial plasmid DNA origin of replication (oriM), an expression cassette containing a mycobacterial promoter, a multiple cloning site, and a transcriptional terminator. For pMV261::HIVgp120, the HIVgp120 gene was fused to the first six codons of the cytoplasmically expressed BCG hsp60 protein. For immunodetection purposes, we fused downstream of the HIV-1 gp120 gene with the influenza virus hemagglutinin epitope (HA) coding sequence and six residues of histidine. The HIV-1 gp120 gene expression was regulated by BCG hsp60 promoter. The primers were designed to incorporate BamHI (forward 5' -CAAGGATCCGAAATTGTGGGTCACAGTC$3^{\prime}$ ) and HindIII (reverse 5'-CAGAAGCTTCTAGTGGTGGTGGTGGT- $3^{\prime}$ ) sites at the $5^{\prime}$ and $3^{\prime}$ termini of the amplified DNA fragment to be cloned into the pMV261 plasmid DNA at 4,326 bp-BamHI/4,346 bp-HindIII.

Plasmid DNA pJH222, a derivative of pMV261, contains also the lys $\mathrm{A}$ complementing gene, under the regulatory control of BCG hsp60 promoter. For the pJH222::HIV-1 gp120 construct, the HIV-1 gp120 gene was fused to the $5^{\prime}$ region encoding the $19 \mathrm{kDa}$ lipoprotein signal sequence from Mycobacterium tuberculosis, and the HA epitope and six residues of histidine were fused downstream. The HIVlgp120 gene expression was under the control of Mycobacteria spp. $\alpha$-antigen promoter. The primers were designed to incorporate HindIII (forward 5'-CAGAAGCTTGGGCCCGAAAAA- $3^{\prime}$ ) and PstI (reverse 5' -CAACTGCAGCTAGTGGTGGT- $3^{\prime}$ ) sites at the $5^{\prime}$ and $3^{\prime}$ termini of the amplified DNA fragment to be cloned into the pJH222 plasmid DNA (335 bp-HindIII/341 bp PstI).
Plasmid DNA pJH223 is an integrative vector (singlecopy, integrated). It contains a DNA cassette encoding kanamycin resistance (Tn903-derived aph gene), an E. coli origin of replication (oriE), a DNA segment carrying the attachment site $(\mathrm{att} P)$ and the integrase (int) gene from the mycobacteriophage L5 [26], the lys A complementing gene under the regulatory control of BCG hsp60 promoter, and an expression cassette containing a mycobacterial promoter, a multiple cloning site and a transcriptional terminator. The pJH223::HIV-1 gp120 plasmid DNA was constructed using the same method as for pJH222::HIV-1 gp120. The primers were designed to incorporate HindIII sites (forward $5^{\prime}$ CAGAAGCTTGGGCCCGAAAAA- $3^{\prime}$ and reverse $5^{\prime}$-CAAAAGCTTCTGCAGCTAGTGGTGG- $3^{\prime}$ ) at the $5^{\prime}$ and $3^{\prime}$ termini of the amplified DNA fragment to be cloned into the pJH223 plasmid DNA (2,134 bp-HindIII).

The correct cloning of the HIV-lgp120 gene into the plasmid DNA and the deletion of the $\sim 900 \mathrm{bp}$ fragment were confirmed by DNA sequencing, PCR and enzyme restriction analysis performed following standard protocols [27].

2.3. Electroporation of Mycobacteria. The BCG wild type host strain was transformed with pMV261::HIV-1 gp120 plasmid DNA and lysine auxotroph of BCG was transformed with pJH222::HIV-1gp120 and pJH223::HIV1gp120 plasmid DNA by electroporation. BCG cultures were grown to an OD of $0.9(600 \mathrm{~nm})$, pelleted at $3,000 \mathrm{rpm}$, washed twice by resuspension and centrifugation $(3,000 \mathrm{rpm})$ in $10 \%$ glycerol at $4^{\circ} \mathrm{C}$ and finally resuspended in $1 / 20$ th of the original culture volume with cold $10 \%$ glycerol. Then $100 \mu \mathrm{L}$ of the cold BCG suspension was mixed with plasmid DNA $(50-500 \mathrm{ng})$ in a 
TABLE 3: Constructs obtained using E. coli/mycobacterial shuttle expression vectors to express HIV-1gp120 antigen (HXBc2).

\begin{tabular}{|c|c|c|c|c|c|}
\hline Constructs & Promoter & Secretion signal & $\begin{array}{l}\text { Resistance } \\
\text { Gene }\end{array}$ & $\begin{array}{l}\text { Complementing } \\
\text { gene }\end{array}$ & Tag \\
\hline pMV261:HIV-1 gp120 & BCG hsp60 & None & Kanamycin & None & $\begin{array}{l}\text { HA } \\
\text { His }\end{array}$ \\
\hline pJH222:HIV-1 gp120 & $\begin{array}{l}\alpha \text {-antigen of } \\
\text { Mycobacterium spp }\end{array}$ & $\begin{array}{l}19 \mathrm{kDa} \text { lipoprotein }(M . \\
\text { tuberculosis) }\end{array}$ & Kanamycin & Lysine & $\begin{array}{l}\text { HA } \\
\text { His }\end{array}$ \\
\hline pJH223:HIV-1 gp120 & $\begin{array}{l}\alpha \text {-antigen of } \\
\text { Mycobacterium spp }\end{array}$ & $\begin{array}{l}19 \mathrm{kDa} \text { lipoprotein }(M . \\
\text { tuberculosis) }\end{array}$ & Kanamycin & Lysine & $\begin{array}{l}\text { HA } \\
\text { His }\end{array}$ \\
\hline
\end{tabular}

HA: hemagglutinin epitope tag from influenza virus; His: Histidine residues.

prechilled $0.2 \mathrm{~cm}$ electroporation cuvette and transformed using the Biorad Gene Pulser electroporator at $2.5 \mathrm{kV}, 25 \mathrm{mF}$, and $1,000 \Omega$. After electroporation $1 \mathrm{ml}$ of $7 \mathrm{H} 9$ medium, supplemented with ADC and containing $0.05 \%$ Tween 80 , was added and incubated at $37^{\circ} \mathrm{C}$ for 12 hours before plating on Middlebrook agar 7H10 medium supplemented with $10 \% \mathrm{ADC}$ and containing $0.05 \%$ Tween 80 and kanamycin $(25 \mu \mathrm{g} / \mathrm{mL})$.

2.4. Detection of the Fragment DNA Deletion. After transformation by electroporation, the rBCG colonies were used as templates for the PCR analysis. Different rBCG colonies were inoculated into $50 \mu \mathrm{L}$ of distilled water, vortexed and $1 \mu \mathrm{L}$ was used for the PCR analysis. Specific primers for cloning the HIV-1 gp120 gene into pMV261, pJH222 and pJH223 vectors were used. The plasmid DNA before BCG transformation (pre-BCG) was used as positive control for the PCR analysis.

2.5. Mycobacterial Plasmid DNA Isolation for DNA Sequencing and Enzyme Restriction Mapping. The QIAprep Spin Miniprep Kit (Qiagen, Hilden, Germany) was used for E. coli and mycobacteria plasmid DNA purification. Different rBCG colonies that showed the DNA fragment deletion by PCR analysis were inoculated in mycobacterial broth culture up to an OD of $0.9(600 \mathrm{~nm})$. This BCG culture was used for mycobacterial plasmid DNA isolation. For mycobacteria, the standard QIAprep Spin Miniprep protocol was used with a slight modification: after resuspension in buffer P1, lysozyme was added at a concentration of $10 \mathrm{mg} / \mathrm{mL}$ and samples were incubated at $37^{\circ} \mathrm{C}$ overnight. For improving the purified plasmid yield, the mycobacterial plasmid DNAs from selected rBCG colonies were transformed in E. coli JM109 using $5 \mu \mathrm{L}$ of the isolated plasmid DNAs, and purified according to the manufacturer's instructions for DNA sequencing and enzyme restriction mapping. The plasmid DNA before BCG transformation (pre-BCG) was used as positive control for the enzyme restriction analysis. The nucleotide sequences were obtained using the BigDye Terminator v3.1 Cycle Sequencing Kit (Applied Biosystems, USA) and ABI PRISM 3100 Genetic Analyzer. Multiple DNA sequence alignments were performed using the Clustal software.
2.6. Western Blot Analysis. BCG transformants were grown to mid-logarithmic phase in liquid 7H9 medium supplemented with 10\% ADC and containing $0.05 \%$ Tween 80 and kanamycin $(25 \mu \mathrm{g} / \mathrm{ml})$. rBCG cultures were centrifuged at $3,000 \mathrm{rpm}$ for 10 minutes at $4^{\circ} \mathrm{C}$. Pellets were washed twice in Phosphate Buffered Saline (PBS) plus $0.02 \%$ Tween 80 and resuspended in $1 \mathrm{ml}$ of extraction buffer $(50 \mathrm{mM}$ Tris$\mathrm{HCl} \mathrm{pH}$ 7.5, $5 \mathrm{mM}$ EDTA, $0.6 \%$ sodium dodecyl sulfate), and added with $5 \mu \mathrm{l}$ of $100 \mathrm{x}$ protease inhibitor cocktail $(1 \mathrm{mg} / \mathrm{ml}$ aprotinin, $1 \mathrm{mg} / \mathrm{ml} \mathrm{E}-64,1 \mathrm{mg} / \mathrm{ml}$ leupeptin, $1 \mathrm{mg} / \mathrm{ml}$ pepstatin A, $50 \mathrm{mg} / \mathrm{ml}$ pefabloc SC, and $10 \mathrm{ml}$ dimethyl sulfoxide). Cells were sonicated for 4 minutes on ice with a Branson sonicator at output control seven and duty cycle of $50 \%$. Extracts were centrifuged at $13,000 \mathrm{rpm}$ for 10 minutes at $4^{\circ} \mathrm{C}$ and supernatants were collected. Proteins were separated on $15 \%$ SDS-polyacrylamide gel. After electroblotting, nitrocellulose membranes were first probed with a 1:1000 dilution of mouse monoclonal antibody HA.11 (Covance, USA) directed to influenza virus hemagglutinin epitope (YPYDVPDYA) followed by a 1:2000 dilution of horseradish peroxidase (HRP)-conjugated antibodies. The membranes were developed using chemoluminiscent ECL (GE Healthcare, USA) as HRP substrate.

\section{Results}

3.1. PCR Detected a 900 bp-DNA Fragment Deletion in the HIV-1gp120 Gene. As shown in Figure 1, PCR analysis of rBCG:HIV-1gp120(pMV261) colonies detected a partial deletion of the HIV-1gp120 DNA coding sequence using the specific primers that were used for cloning the DNA sequence into the pMV261 vector. This deletion was reproduced in 12 out of 14 rBCG:HIV-1 gp120 (pMV261) colonies analyzed (data not shown). In addition, this partial deletion was observed only by PCR of plasmid DNA from BCG colonies in which the HIV-1gp120 DNA fragment was inserted into the pMV261 vector and the gene expression was regulated by the hsp60 promoter from BCG (Figure 1, lane 2). The deletion was not detected when the DNA fragment encoding the HIVlgp120 antigen was cloned into pJH222 and pJH223 vectors under Mycobacteria spp. $\alpha$-antigen promoter regulation (Figure 1, lanes 4 and 6). The deletion was not detected in all 10 rBCG:HIV-1gp120 (pJH222) colonies screened by PCR (data not shown). 


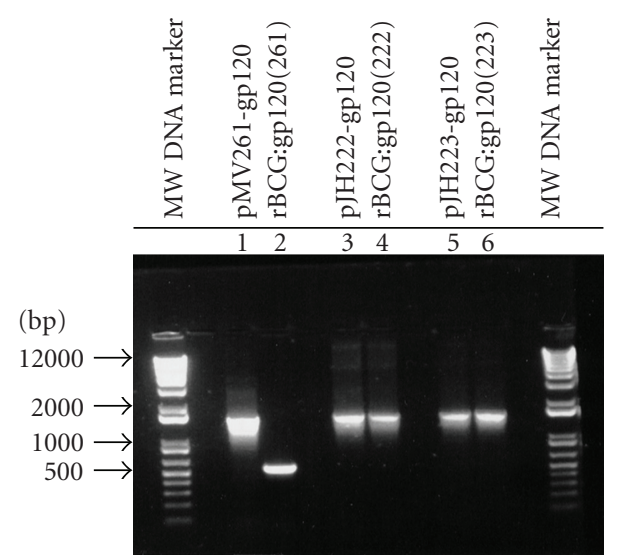

FIgUre 1: PCR analysis of the HIV-1gp120 gene cloned into pMV261, pJH222 and pJH223 vectors. Lane 1, 3 and 5 are PCR products of the HIV-1gp120 gene (1578 bp) cloned into pMV261 (lane 1), pJH222 (lane 3) and pJH223 (lane 5) vectors, respectively. Plasmid DNA before BCG transformation (pre-BCG) were used as positive controls (lane 1, 3 and 5). Lane 2, 4 and 6 are PCR products from the rBCG:HIV-1gp120 (pMV261), rBCG:HIV1gp120 (pJH222) and rBCG:HIV-1gp120 (pJH223) colonies.

3.2. Enzymatic Restriction Analysis Revealed a $9900 \mathrm{bp}$ DNA Fragment Deletion. We also compared the restriction enzyme digestion profile of the pMV261 and pJH222 plasmid DNA vectors with and without heterologous HIVlgp120 DNA insert, pre- and post-BCG transformation (Figure 2). The pMV261 plasmid DNA vector without the DNA insert corresponding to HIV-1gp120 gene did not show any difference in the enzymatic restriction profiles from preand post-BCG transformations (Figure 2, lanes 1 and 2). The same result was obtained for the pJH222 plasmid DNA vector containing the DNA insert (Figure 2, lanes 5 and 6). In contrast, the pMV261 containing the HIV-1gp120 insert showed different enzymatic restriction profiles from pre- and post-BCG transformations (Figure 2, lanes 3 and 4).

\subsection{The $\sim 900 \mathrm{bp}$-Partial DNA Fragment Deletion Corresponds} to the HIV-1gp120 Core. Following sequence analysis and molecular characterization of the deleted DNA fragment in the 10 rBCG:HIV-1gp120 (pMV261) mutant colonies, we demonstrated that the partial deletion ( $\sim 900 \mathrm{bp})$ corresponds to gp 120 core rich in potential glycosylation sites and the variable regions V3, V4, and V5 of HIV-1 env, a key structure involved in the HIV union to its receptor (CD4) and coreceptor on the host cell surface. Strikingly, the deleted DNA fragment in the rBCG mutants contained the DNA sequence coding for HIV-1 envelope immunodominant CTL, murine $\mathrm{H}-2 \mathrm{D}^{\mathrm{d}}$-restricted epitope P18-I10 (Figure 3). Deletion in HIV-1gp120 gene was not observed in any rBCG:HIV1gp120 (pJH222) and rBCG:HIV1gp120 (pJH223) colonies that carry the pJH222:HIV-1gp120 and pJH223:HIV1gp120 vectors containing the Mycobacteria spp. $\alpha$-antigen promoter to regulate the HIV1gp120 gene expression, indicating that

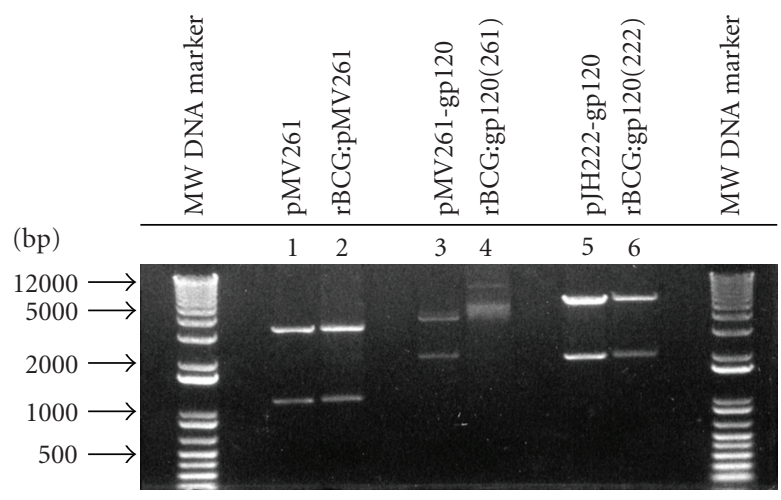

FIGURE 2: Enzymatic restriction analysis of pMV261::HIV-1gp120 and pJH222::HIV-1gp120 plasmid DNA after BCG transformation. Lane 1, pMV261 plasmid DNA without HIV-1 gp120 DNA insert digested with BglII; Lane 2 pMV261 plasmid DNA without HIV1 gp120 DNA insert from BCG colonies, digested with BglII, Lane 3, pMV261-HIV-1gp120 plasmid DNA digested with SacII; lane 4, pMV261-HIV-1gp120 plasmid DNA from rBCG:HIV-1gp120 colonies, digested with SacII; lane 5, pJH222-HIV-1gp120 plasmid DNA digested with HpaI and lane 6, pJH222-HIV-1gp120 plasmid DNA from rBCG:HIV-1 gp120 colonies, digested with HpaI. Plasmid DNAs before BCG transformation (pre-BCG) were used as positive controls (lane 1, 3 and 5).

the deletions were not due solely to the toxic effects of HIVlgp120 expression during growth of the recombinant BCG strains.

3.4. A Truncated Protein Was Expressed in rBCG Mutants That Show the $\sim 900 \mathrm{bp}$-DNA Fragment Deletion. Expression of HIV-1gp120 protein by rBCG:HIV-1gp120 (pMV261 and pJH222) strains was confirmed by SDS-PAGE and western blot analysis of whole-cell BCG lysates. The chimeric $19 \mathrm{kDa}$ lipoprotein signal sequence-HIV-1gp120-Flu-His recombinant protein, with a relative molecular mass $(\mathrm{Mr})$ of 67 kiloDalton $(\mathrm{kDa})$ was present in lysates of rBCG:HIV1gp120 (pJH222) bacterial cells containing the 1.82 kilobasepair (kbp) HIV-1gp120 DNA coding sequence in pJH222 vector. The apparent Mr of HIV-1gp120 protein was consistent with that predicted by the gene sequence without posttranslation modification. By contrast, when all rBCG:HIV1gp120 (pMV261) mutant colonies were analyzed by western blot, we detected a band of $28 \mathrm{kDa}$ (truncated protein) instead of the expected $67 \mathrm{kDa}$ protein (Figure 4).

\section{Discussion}

Mycobacterium tuberculosis infection is a major cause of human morbidity and mortality, and vaccine is the most cost-effective intervention to prevent disease. Mycobacterium bovis BCG is a widely used vaccine against tuberculosis, and a single dose given at birth confers long-lasting immunity. Mycobacterium bovis BCG has been suggested as an ideal delivery system for expression of foreign antigens due to the long persistence of BCG in the immunized host. Thus, Mycobacterium bovis BCG not only is used as vaccine against 


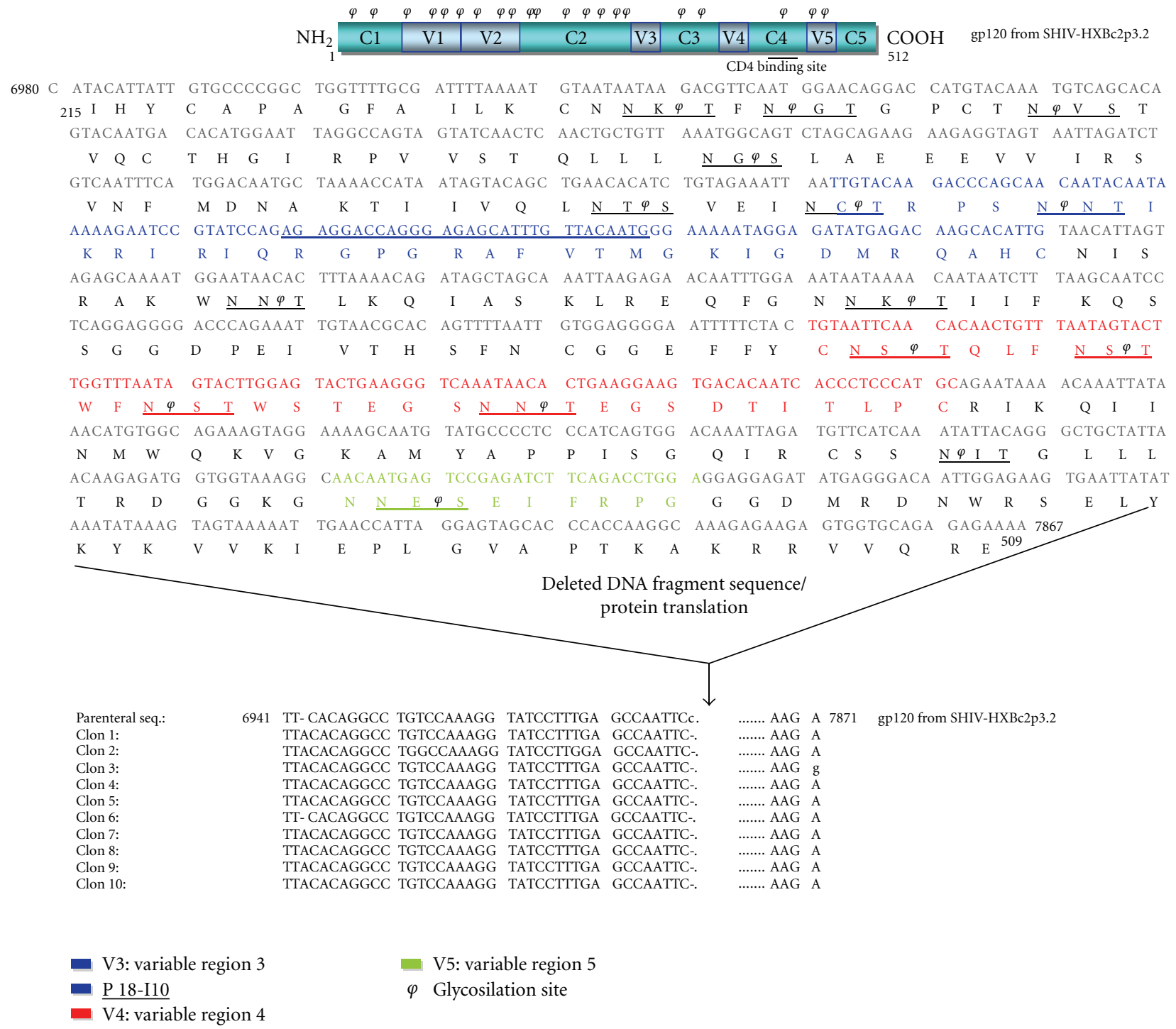

Figure 3: Multiple HIV-1gp120 DNA sequence alignment carrying the 900-bp deletion from the mutant rBCG:HIV-1gp120 (pMV261) colonies. HIV-1gp120 DNA sequences of 10 mutant rBCG:HIV-1gp120 (pMV261) colonies were aligned and compared with the parental nucleotide sequence of the HIV-1gp120 gene. The 900 bp deleted fragment (sequence and position shown in the scheme) structurally corresponds to the gp120 core (see protein translation).

tuberculosis, but also offers great potential for innovative approaches for development of polyvalent vaccines [25, 28$30]$. In vivo genetic stability and persistence are of special importance for the use of live bacterial vaccines. A mutant BCG which is rapidly eliminated by the host immune system is unlikely to be an effective vaccine [31]. The rearrangement of DNA is one of the fundamental properties of life, including both prokaryotes and eukaryotes, with great biological significance. Some of these DNA rearrangements are important in controlling gene expression in specific cell types; others may play an evolutionary role by contributing to genetic diversity.

Some studies using episomal vectors in $\mathrm{rBCG}$ reported high levels of stability in vitro. For example, plasmids responsible for expressing $M$. Tuberculosis $\alpha$-antigen in BCG were stable over six consecutive 4-week cultures without selection and changes in the level of expression [32]. An $\alpha$-antigen-HIV-1 V3J1 chimeric protein was secreted by rBCG for at least 450 passages in vitro [33]. However, in vitro instability of episomal vectors has also been reported regularly. Lim et al. [34] described that expression of SIV mac 251 env (aminoacids, aa, 1-245) by rBCG was stable in culture ( $\mathrm{pBlaF}$ promoter), but rBCG harboring the same vector containing aa $1-521$ or aa $215-521$ were unstable. Chawla and Das Gupta [35] reported that the disruption of pAL5000-derived vectors in M.smegmatis through transposition of an insertion sequence upstream of the kanamycin resistance gene led to structural instability and large deletions. Kumar et al. studied the stability of the expression of Escherichia coli $\beta$-galactosidase under the 


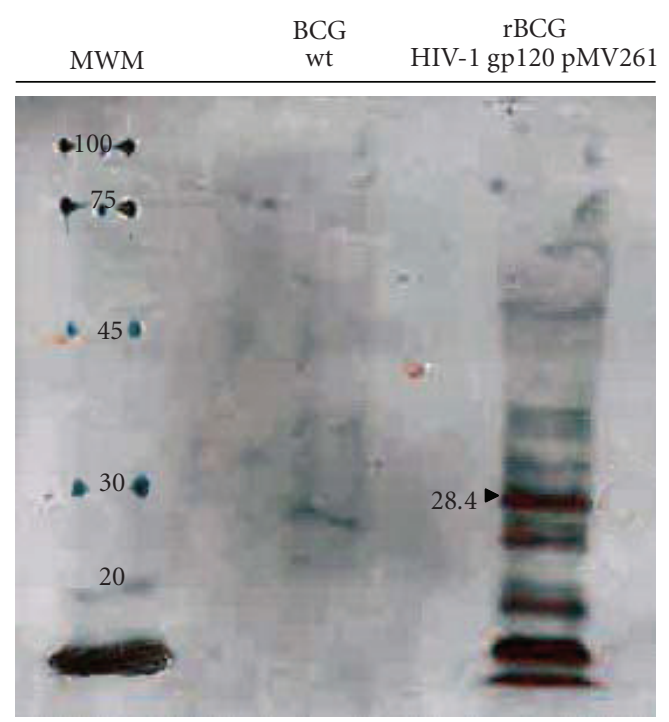

(a)

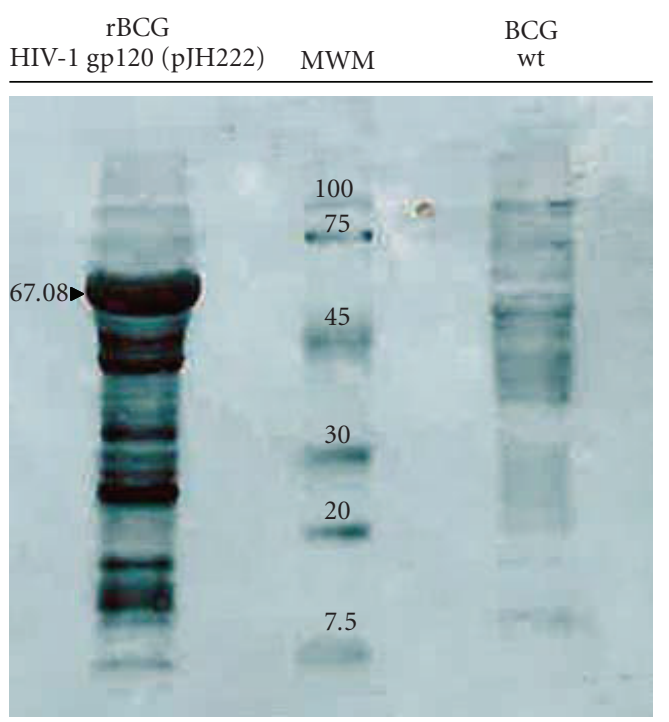

(b)

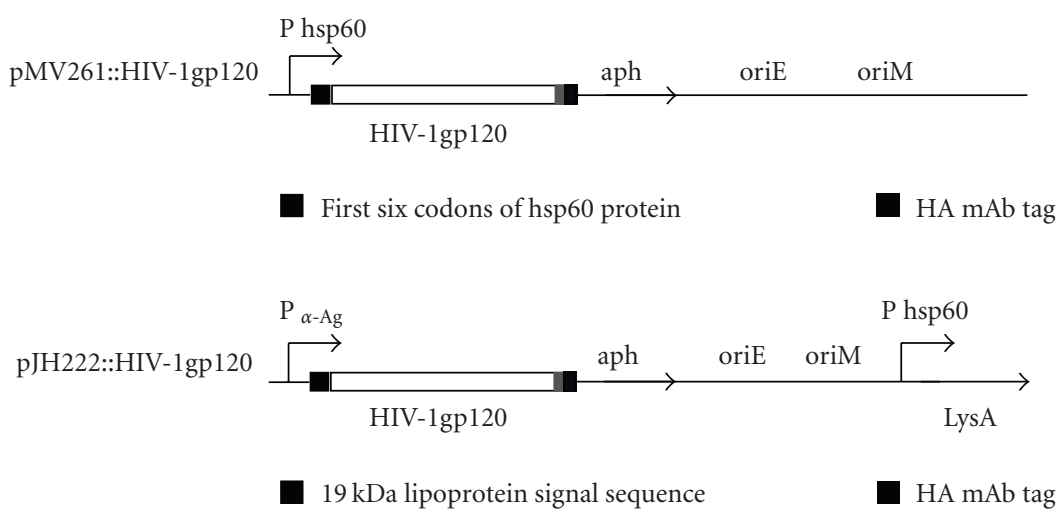

(c)

FIGURE 4: Expression of HIV-1gp120 protein in rBCG:HIV-1gp120 (pMV261) and rBCG:HIV-1gp120 (pJH222) strains by Western blot analysis of whole-cell BCG lysates. (a) A band of $\sim 28 \mathrm{kDa}$ was detected when analizing the rBCG:HIV-1gp120 (pMV261) colonies. (b) A $67 \mathrm{kDa}$ protein was detected in lysates of rBCG:HIV-1gp120 (pJH222) bacterial cells containing the $1.82 \mathrm{kilobase}(\mathrm{kb}) \mathrm{HIV}-1 \mathrm{gp} 120$ DNA coding sequence into pJH222 vector. The gp120 expression was determined using anti-HA MAb. Not transformed BCG (BCG wild type) were utilized as negative controls. (c) The HIV-1gp120 (HXBc2) gene was cloned into the E.coli/mycobacteria shuttle plasmids pMV261 and pJH222 (both multicopy and episomal). The gp120 env in the plasmids is under the control of BCG hsp60 promoter (P hsp60) and M.Tuberculosis $\alpha$-antigen promoter ( $\mathrm{P} \alpha$-Ag). Both plasmids contained kanamycin resistance gene (aph) and an E.coli origin of replication (ori E) and a mycobacterial origin of replication (oriM). In addition, pJH222 contained the complementing lysA gene. The HIV-1gp120 gene was fused to the first six codons of BCG hsp60 (pMV261) or fused to M. tuberculosis 19-kDa lipoprotein signal sequence (pJH222). MWM, molecular weight markers.

control of hsp60 promoter in Mycobacterium smegmatis using integrative and replicative vectors. In the case of replicative vectors, they detected higher frequencies of lacZ expression inactivation as compared with the same lac $Z$ cassette, when present in integrated state [22]. However, Springer et al. [36] reported a high-frequency loss of pMV361-based integrating vectors from $M$. smegmatis and BCG. Al-Zarouni and Dale investigated the effects of various combinations of posttranslational signals and promoters on heterologous gene expression and stability in different BCG strains. Plasmid DNAs were constructed using mycobacterial promoters (hsp60, 19-kDa antigen,
85A antigen, from Mycobacterium tuberculosis complex, and the $18-\mathrm{kDa}$ antigen from Mycobacterium leprae) and post-translation signals (85A antigen secretion and $19-\mathrm{kDa}$ antigen acylation signals), coupled with reporter genes. They provided evidence that the $85 \mathrm{~A}$ secretion signal markedly enhanced the levels of cell-associated product, while the 19$\mathrm{kDa}$ acylation signal had little effect on gene expression. This group also demonstrated that the hsp60 promoter caused plasmid DNA instability and showed that various deletions in the promoter region occurred during or soon after transformation, but not during subsequent growth of the transformants, nor with other promoters [37]. Gross 
instability was also observed when the episomal vectors were used to express rotavirus VP6 protein under the control of hsp60 promoter [38]. The possibility of inserting foreign genes into the chromosome at precise positions to ensure the persistence of the heterologous genetic information in the recombinant vaccine strains would represent a crucial step in the development of Mycobacterium bovis BCG as a live vaccine vector for expression of heterologous antigens $[28,39]$. Even though integrative plasmid DNAs have been shown to be more stable as they are incorporated into the chromosome via homologous recombination, Baulard and colleagues demonstrated that with replicating plasmid DNAs, relatively high levels of homologous recombination were obtained in fast- and slow-growing mycobacteria, and $100 \%$ of the selected clones underwent homologous recombination [40].

Thus, if BCG is to be used as a live bacterial carrier for generating novel vaccine candidates and as an immunotherapeutic agent, it is essential that a more genetically stable strain be developed. Sander and collaborators demonstrated that Mycobacterium bovis BCG recA mutants are a valuable tool for the further development of BCG as an antigen delivery system to express foreign antigens and as a source of a genetically stable vaccine against tuberculosis [41]. This was confirmed in a study performed by Keller and coworkers where it was observed that $r e c A$ inactivation in BCG Russia strain was in part responsible for its high degree of genomic stability, resulting in a substrain that has fewer genetic alterations than other vaccine substrains with respect to $M$. bovis AF2122/07 wild-type [42].

Expression of HIV genes in BCG is not always possible. Lack of success in this respect may be due to overexpression of lethality or other forms of protein toxicity. Stover et al. were unable to express HIV-1 gp120 (hsp60 promoter) from an episomal vector, but could express it from an integrative vector $[19,25]$.

In our study, we have observed that, in the case of the replicative pMV261 vector, disruption of HIV-1gp120 gene expression was due to a consensus DNA fragment deletion in the HIV1gp120 gene detected in all ten different BCG clones. By contrast, we did not detect any HIV-1gp120 gene expression disruption in the replicative (pJH222) and integrative (pJH223) vectors. Sequence analysis indicated that the DNA fragment deletion contained several potential glycosylation sites, the variable regions V3, V4 and V5 and a CTL immunodominant epitope (P18-I10 peptide). Based on this observation, we hypothesized that the loss of expression of this immunodominant epitope could help mycobacteria to escape the host's immunological response and may represent part of an ongoing adaptation to survival in host environments that are screened by immunological defense mechanisms. Huber and collaborators have described a natural loss of expression of highly immunogenic proteins caused by a variety of genomic changes in Mycobacterium ulcerans that may confer a selective advantage to this emerging pathogen [43]. On the other hand, the deleted DNA sequence in the rBCG:HIV-1gp120(pMV261) mutant colonies analyzed in our study $(n=10)$ was highly conserved, which is in disagreement with Dennehy et al. that deletions in the VP6 heterologous gene (hsp60 promoter) in rBCG were random and different among clones examined [38].

We have demonstrated that the use of weak promoters (Mycobacteria spp. $\alpha$-antigen promoter) to regulate HIVlgp120 gene expression and BCG lysine auxotrophs complemented with a lysine gene do, in fact, prevent the disruption of gene expression caused by genetic rearrangements. The results showed that such genetic rearrangements can significantly affect the expression of foreign genes in mycobacteria. Thus, it could result in an rBCG that does not induce optimal immune responses to the HIV antigen.

Mycobacterium bovis Bacillus Calmette-Guérin (BCG) as a live vector of recombinant bacterial vaccine is a promising system to be used. However, few promising approaches have emerged in the last five years to overcome the genetic instability in rBCG-based vaccines. To prevent the plasmid instability in vivo and in vitro and the genetic rearrangement by mycobacteria, different approaches should be considered: (i) the use of expression vectors containing small HIV DNA coding sequences, (ii) DNA fragments lacking glycosilation sites, (iii) the use of weak promoters, (iv) the use of BCG auxotrophic strains (containing the complementing gene in the expression vectors), (v) the use of inducible promoters, (vi) codon optimization of the recombinant gene, (vii) the choice of expression vector backbone and (viii) antigen secretion to enhance the immunogenicity and to prevent foreign proteins from becoming toxic to BCG.

In five years' time we will have a better picture of how the immune responses induced by rBCG could be enhanced, and what sort of route, doses, immunization schedule, timing for immunological assays are likely to induce optimal responses in mice and monkeys. Heterologous prime-boost regimen, different immunization schedule, routes and doses, should be performed to evaluate the potential for enhancing specific immune responses.

Mycobacterium Bovis BCG is a promising approach as a bacterial live recombinant vaccine vehicle. BCG has a long record of safe use in humans and is able to induce longlasting immunity. In addition, it could be the best hope for protecting newborn infants, reducing the adult burden of HIV infection and protecting neonates against vertical transmission.

\section{Acknowledgments}

The authors are grateful to Dr. Barry R. Bloom and Dr. William R. Jacobs for providing the plasmid DNA pJH222 and pJH223 and BCG wild type and lysine auxotroph of BCG for research purposes. This work has been supported by HIVACAT, the Foundation for Research and Prevention of AIDS in Spain (FIPSE 36338/02), Fundación Mutua Madrileña de Automóviles (second call for proposals), and Fundació BCN SIDA 2002. N.S. is a Red Temática de Investigación Cooperativa en SIDA (RIS) Senior Fellow. Raquel Fernández-Lloris is an EDCTP Research Fellow. 


\section{References}

[1] Children on the Brink, A Population, Health and Nutrition Information Project under USAID contract no. HRN-C00-00-0004-00. The Joint United Nations Programme on HIV/AIDS (UNAIDS), the United Nations Children's Fund (UNICEF), and the United States Agency for International Development (USAID). United Nations Children's Fund, 3 United Nations Plaza, New York, NY, USA, 4th edition, 2004.

[2] AIDS Epidemic Update, Joint United Nations Programme on HIV/AIDS (UNAIDS). UNAIDS, Geneva, Switzerland, November 2009.

[3] M. Kawada, T. Tsukamoto, H. Yamamoto et al., "Gag-specific cytotoxic T-lymphocyte-based control of primary simian immunodeficiency virus replication in a vaccine trial," Journal of Virology, vol. 82, no. 20, pp. 10199-10206, 2008.

[4] T. Matano, M. Kobayashi, H. Igarashi et al., "Cytotoxic T lymphocyte-based control of simian immunodeficiency virus replication in a preclinical AIDS vaccine trial," Journal of Experimental Medicine, vol. 199, no. 12, pp. 1709-1718, 2004.

[5] J. E. Schmitz, M. J. Kuroda, S. Santra et al., "Control of viremia in simian immunodeficiency virus infection by $\mathrm{CD}^{+}$ lymphocytes," Science, vol. 283, no. 5403, pp. 857-860, 1999.

[6] E. S. Rosenberg, J. M. Billingsley, A. M. Caliendo et al., "Vigorous HIV-1-specific $\mathrm{CD}^{+}{ }^{+} \mathrm{T}$ cell responses associated with control of viremia," Science, vol. 278, no. 5342, pp. 14471450, 1997.

[7] N. L. Letvin, "Strategies for an HIV vaccine," Journal of Clinical Investigation, vol. 110, no. 1, pp. 15-20, 2002.

[8] J. Joseph, N. Saubi, E. Pezzat, and J. M. Gatell, "Progress towards an HIV vaccine based on recombinant Bacillus Calmette-Guérin: failures and challenges," Expert Review of Vaccines, vol. 5, no. 6, pp. 827-838, 2006.

[9] N. Ohara and T. Yamada, "Recombinant BCG vaccines," Vaccine, vol. 19, no. 30, pp. 4089-4098, 2001.

[10] E.-J. Im, N. Saubi, G. Virgili et al., "Vaccine platform for prevention of tuberculosis and mother-to-child transmission of human immunodeficiency virus type 1 through breastfeeding," Journal of Virology, vol. 81, no. 17, pp. 9408-9418, 2007.

[11] J. D. Cirillo, C. K. Stover, B. R. Bloom, W. R. Jacobs Jr., and R. G. Barletta, "Bacterial vaccine vectors and bacillus CalmetteGuérin,” Clinical Infectious Diseases, vol. 20, no. 4, pp. 10011009, 1995.

[12] P. Nevers and H. Saedler, "Transposable genetic elements as agents of gene instability and chromosomal rearrangements," Nature, vol. 268, no. 5616, pp. 109-115, 1977.

[13] T. D. Petes and C. W. Hill, "Recombination between repeated genes in microorganisms," Annual Review of Genetics, vol. 22, pp. 147-168, 1988.

[14] H. J. Reif and H. Saedler, "IS1 is involved in deletion formation in the gal region of E. coli K12," Molecular and General Genetics, vol. 137, no. 1, pp. 17-28, 1975.

[15] J. R. Roth, N. Benson, T. Galitski, K. Haack, J.G. Lawrence, and L. Miesel, "Rearrangements of the bacterial chromosome formation and applications," in Escherichia coli and Salmonella typhimurium, F. C. Neidhardt, R. Curtiss, J. L. Ingraham, et al., Eds., pp. 2256-2276, ASM Press, Washington, DC, USA, 1996.

[16] Z. Piao, K. Shibayama, S. Mori, J.-I. Wachino, and Y. Arakawa, "A novel insertion sequence, IS1642, of Mycobacterium avium, which forms long direct repeats of variable length: research letter," FEMS Microbiology Letters, vol. 291, no. 2, pp. 216-221, 2009.
[17] A. Coros, E. DeConno, and K. M. Derbyshire, "IS6110, a Mycobacterium tuberculosis complex-specific insertion sequence, is also present in the genome of Mycobacterium smegmatis, suggestive of lateral gene transfer among mycobacterial species," Journal of Bacteriology, vol. 190, no. 9, pp. 34083410, 2008.

[18] X.-M. Wang, A. Galamba, D. F. Warner et al., "IS1096mediated DNA rearrangements play a key role in genome evolution of Mycobacterium smegmatis," Tuberculosis, vol. 88, no. 5, pp. 399-409, 2008.

[19] M. Dennehy and A.-L. Williamson, "Factors influencing the immune response to foreign antigen expressed in recombinant BCG vaccines," Vaccine, vol. 23, no. 10, pp. 1209-1224, 2005.

[20] R. N. Husson, B. E. James, and R. A. Young, "Gene replacement and expression of foreign DNA in mycobacteria," Journal of Bacteriology, vol. 172, no. 2, pp. 519-524, 1990.

[21] F. Haeseleer, "Structural instability of recombinant plasmids in mycobacteria," Research in Microbiology, vol. 145, no. 9, pp. 683-687, 1994.

[22] D. Kumar, B. S. Srivastava, and R. Srivastava, "Genetic rearrangements leading to disruption of heterologous gene expression in mycobacteria: an observation with Escherichia coli $\beta$-galactosidase in Mycobacterium smegmatis and its implication in vaccine development," Vaccine, vol. 16, no. 1112, pp. 1212-1215, 1998.

[23] M. Gheorghiu, P. H. Lagrange, and C. Fillastre, "The stability and immunogenicity of a dispersed-grown freeze-dried Pasteur BCG vaccine," Journal of Biological Standardization, vol. 16, no. 1, pp. 15-26, 1988.

[24] M. S. Pavelka Jr and W. R. Jacobs Jr, "Comparison of the construction of unmarked deletion mutations in Mycobacterium smegmatis, Mycobacterium bovis bacillus Calmette-Guérin, and Mycobacterium tuberculosis H37Rv by allelic exchange," Journal of Bacteriology, vol. 181, no. 16, pp. 4780-4789, 1999.

[25] C. K. Stover, V. F. de la Cruz, T. R. Fuerst et al., "New use of BCG for recombinant vaccines," Nature, vol. 351, no. 6326, pp. 456-460, 1991.

[26] M. H. Lee, L. Pascopella, W. R. Jacobs Jr., and G. F. Hatfull, "Site-specific integration of mycobacteriophage L5: integration-proficient vectors for Mycobacterium smegmatis, Mycobacterium tuberculosis, and bacille Calmette-Guérin," Proceedings of the National Academy of Sciences of the United States of America, vol. 88, no. 8, pp. 3111-3115, 1991.

[27] F. M. Ausubel, R. Brent, and R. E. Kingston, Eds., Current Protocols in Molecular Biology, John Wiley \& Sons, New-York, NY, USA, 2000-2010.

[28] A. Aldovini and R. A. Young, "Humoral and cell-mediated immune responses to live recombinant BCG-HIV vaccines," Nature, vol. 351, no. 6326, pp. 479-482, 1991.

[29] K. Matsuo, R. Yamaguchi, A. Yamazaki et al., "Establishment of a foreign antigen secretion system in mycobacteria," Infection and Immunity, vol. 58, no. 12, pp. 4049-4054, 1990.

[30] N. Winter, M. Lagranderie, J. Rauzier et al., "Expression of heterologous genes in Mycobacterium bovis BCG: induction of a cellular response against HIV-1 Nef protein," Gene, vol. 109, no. 1, pp. 47-54, 1991.

[31] B. R. Bloom and P. E. M. Fine, "The BCG experience: implications for future vaccines against tuberculosis," in Tuberculosis: Pathogenesis, Protection, and Control, B. R. Bloom, Ed., pp. 531-558, ASM Press, Washington, DC, USA, 1994.

[32] M. A. Horwitz, G. Harth, B. J. Dillon, and S. MaslesaGalic, "Recombinant bacillus Calmette-Guérin (BCG) vaccines expressing the Mycobacterium tuberculosis $30-\mathrm{kDa}$ major secretory protein induce greater protective immunity against 
tuberculosis than conventional BCG vaccines in a highly susceptible animal model," Proceedings of the National Academy of Sciences of the United States of America, vol. 97, no. 25, pp. 13853-13858, 2000.

[33] M. Kawahara, K. Matsuo, T. Nakasone et al., "Combined intrarectal/intradermal inoculation of recombinant Mycobacterium bovis bacillus Calmette-Guérin (BCG) induces enhanced immune responses against the inserted HIV-1 V3 antigen," Vaccine, vol. 21, no. 3-4, pp. 158-166, 2002.

[34] E. M. Lim, M. Lagranderie, R. Le Grand et al., "Recombinant Mycobacterium bovis BCG producing the N-terminal half of SIVmac251 Env antigen induces neutralizing antibodies and cytotoxic T lymphocyte responses in mice and guinea pigs," AIDS Research and Human Retroviruses, vol. 13, no. 18, pp. 1573-1581, 1997.

[35] M. Chawla and S. K. Das Gupta, "Transposition-induced structural instability of Escherichia coli-mycobacteria shuttle vectors," Plasmid, vol. 41, no. 2, pp. 135-140, 1999.

[36] B. Springer, P. Sander, L. Sedlacek, K. Ellrott, and E. C. Böttger, "Instability and site-specific excision of integrationproficient mycobacteriophage L5 plasmids: development of stably maintained integrative vectors," International Journal of Medical Microbiology, vol. 290, no. 8, pp. 669-675, 2001.

[37] M. Al-Zarouni and J. W. Dale, "Expression of foreign genes in Mycobacterium bovis BCG strains using different promoters reveals instability of the hsp60 promoter for expression of foreign genes in Mycobacterium bovis BCG strains," Tuberculosis, vol. 82, no. 6, pp. 283-291, 2002.

[38] M. Dennehy, W. Bourn, D. Steele, and A.-L. Williamson, "Evaluation of recombinant BCG expressing rotavirus VP6 as an anti-rotavirus vaccine," Vaccine, vol. 25 , no. 18, pp. 36463657, 2007.

[39] T. R. Fuerst, C. K. Stover, and V. F. de la Cruz, "Development of BCG as a live recombinant vector system: potential use as an HIV vaccine," Biotechnology Therapeutics, vol. 2, no. 1-2, pp. 159-178, 1991.

[40] A. Baulard, L. Kremer, and C. Locht, "Efficient homologous recombination in fast-growing and slow-growing mycobacteria," Journal of Bacteriology, vol. 178, no. 11, pp. 3091-3098, 1996.

[41] P. Sander, K. G. Papavinasasundaram, T. Dick et al., "Mycobacterium bovis BCG recA deletion mutant shows increased susceptibility to DNA-damaging agents but wild-type survival in a mouse infection model," Infection and Immunity, vol. 69, no. 6, pp. 3562-3568, 2001.

[42] P. M. Keller, E. C. Böttger, and P. Sander, "Tuberculosis vaccine strain Mycobacterium bovis BCG Russia is a natural recA mutant," BMC Microbiology, vol. 8, article 120, 2008.

[43] C. A. Huber, M.-T. Ruf, G. Pluschke, and M. Käser, "Independent loss of immunogenic proteins in Mycobacterium ulcerans suggests immune evasion," Clinical and Vaccine Immunology, vol. 15, no. 4, pp. 598-606, 2008. 

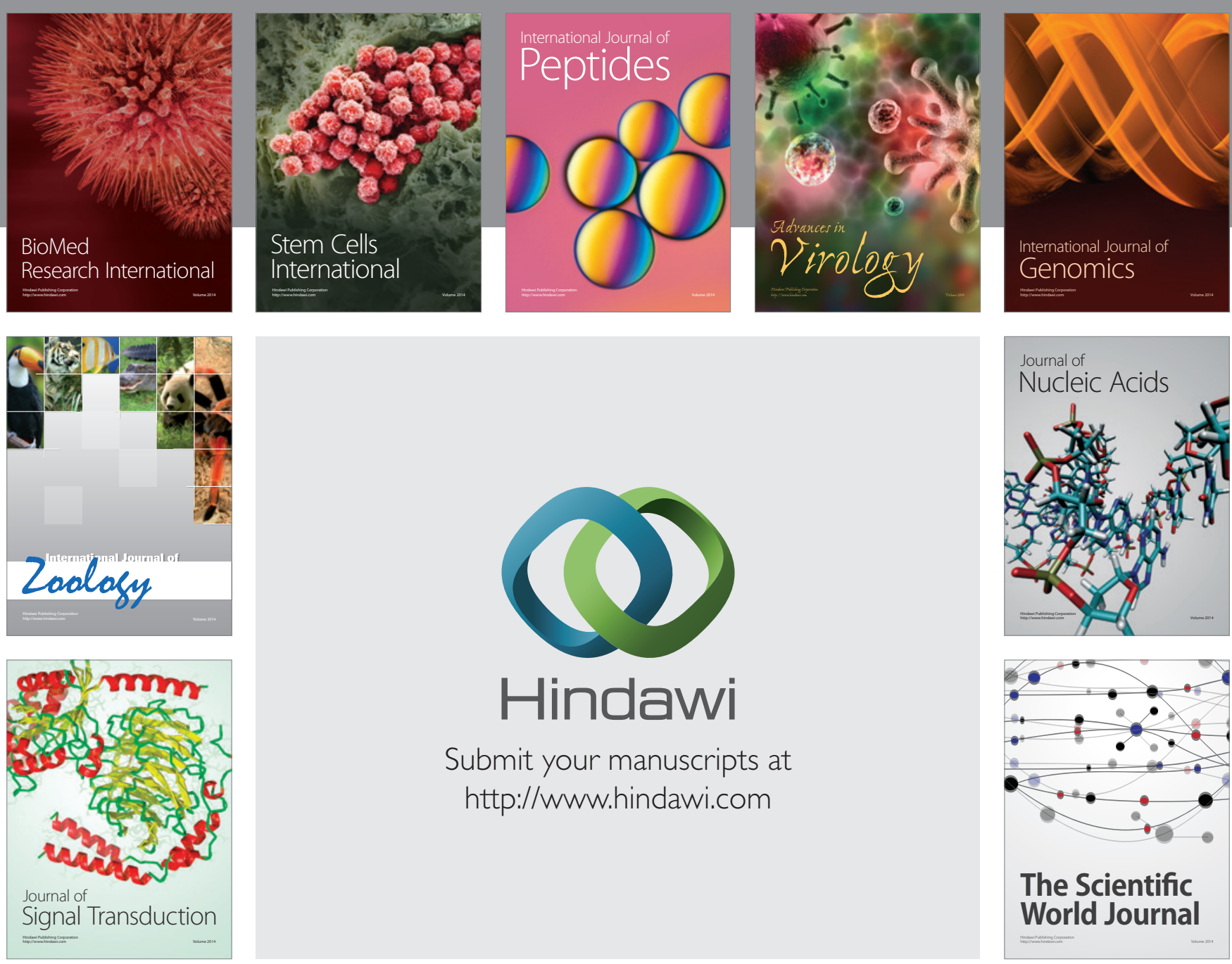

Submit your manuscripts at

http://www.hindawi.com
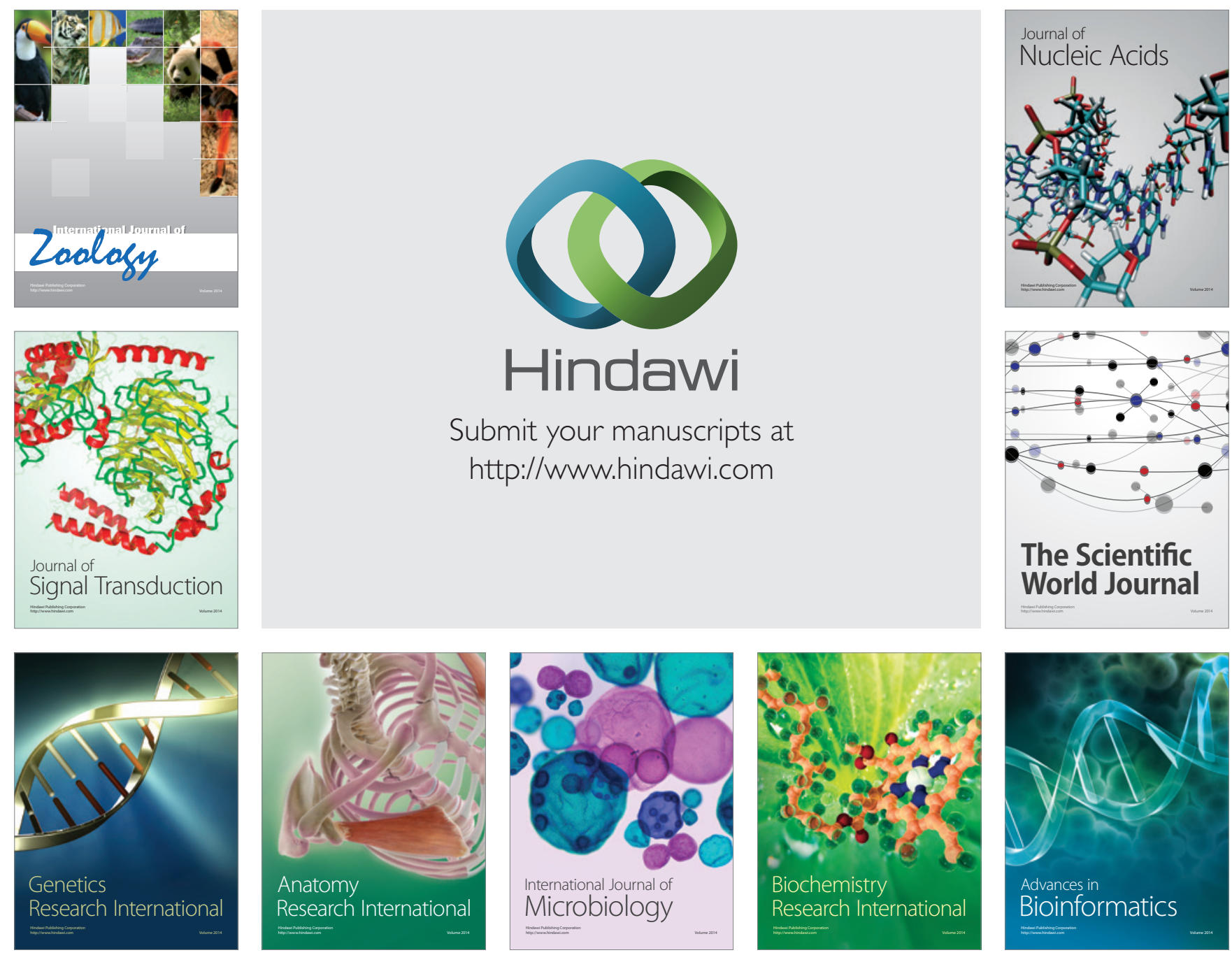

The Scientific World Journal
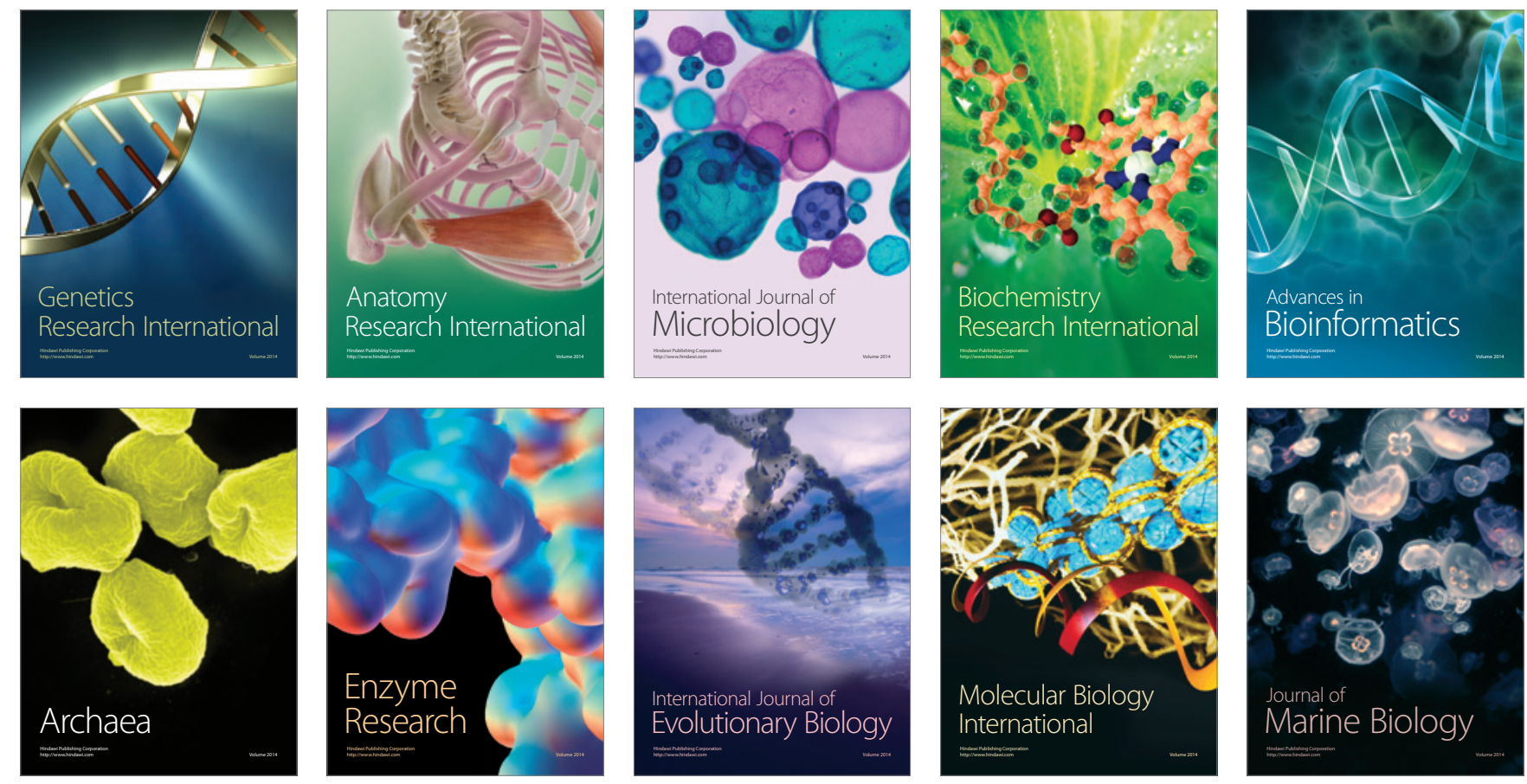